\title{
Silicon Oxycarbide Platform for Integrated Photonics
}

\author{
Faisal Ahmed Memon, Francesco Morichetti, Matteo Cantoni, Claudio Somaschini, Marco Asa, \\ Riccardo Bertacco, Bhawani Shankar Chowdhry and Andrea Melloni
}

\begin{abstract}
In this paper we present silicon oxycarbide (SiOC) as a potential dielectric platform for integrated photonic applications. By controlling the amount of carbon and oxygen in the composition of SiOC films, which in this work are deposited by using a reactive $R F$ magnetron sputtering process, the refractive index of the material can be widely tuned from less than that of silica 1.40 to almost that of silicon carbide (3.0), while keeping high transparency in the near-infrared wavelength range. An indepth analysis of the structural, morphological and optical properties of the deposited $\mathrm{SiOC}$ thin films is reported, pointing out the relationship between the change in the film composition and the tuneability of the refractive index. Single mode optical waveguides with a refractive index contrast of up to $28 \%$ and propagation loss as low as $2 \mathrm{~dB} / \mathrm{cm}$ at $1550 \mathrm{~nm}$ are demonstrated. An integrated Mach-Zehnder interferometer is presented as a first example of PIC realized on high refractive index SiOC platform. Results show that $\mathrm{SiOC}$ is a promising alternative to conventional dielectric platforms for the realization of PICs.
\end{abstract}

Index Terms - integrated optics, optical materials, optical waveguides, silicon oxycarbide.

\section{INTRODUCTION}

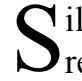
ilicon oxycarbide ( $\mathrm{SiOC})$ is a glass compound that has received significant attention from the scientific community and has already found applications in several fields of technologies, such as ion batteries and low- $k$ interlayer dielectrics [1-10]. Recently, we demonstrated that $\mathrm{SiOC}$ has also promising optical characteristics to become an

Manuscript received ...

The research leading to these results received funding from the European Union's H2020 Programme Super-Pixels (grant 829116) and by Fondazione Cariplo Project "Advanced Control Technologies for Integrated Optics (ACTIO)", Rif. 2016-0881. F.A.M. acknowledges Erasmus Mundus LEADERS Project for the Ph.D. scholarship.

F. A. Memon is with the Department of Telecommunications Engineering, Mehran University of Engineering \& Technology, Jamshoro 76062, Pakistan (email: faisal.memon@faculty.muet.edu.pk)

F. Morichetti and A. Melloni are with the Dipartimento di Elettronica, Informazione e Bioingegneria, Politecnico di Milano, Piazza Leonardo da Vinci, 32 - 20133, Milano, Italy (email: francesco.morichetti@polimi.it; andrea.melloni@polimi.it)

M.Cantoni, M.Asa, R.Bertacco are with Dipartimento di Fisica, Politecnico di Milano, Piazza Leonardo da Vinci, 32-20133, Milano, Italy (email: matteo.cantoni@polimi.it; marco.asa@polimi.it; riccardo.bertacco@polimi.it)

C. Somaschini is with Polifab, Politecnico di Milano, Via G. Colombo, 8120133 Milano, Italy (email: claudio1.somaschini@polimi.it)

B.S. Chowdhry is Professor Emeritus and Distinguished National Professor at Mehran University of Engineering \& Technology, Jamshoro 76062, Pakistan (bhawani.chowdhry@,faculty.muet.edu.pk)

Color versions of one or more of the figures in this paper are available online at http://ieeexplore.iee.org. enabling material for photonic integrated circuits [11], because of its widely tuneable refractive index and high thermo-optic coefficient, the latter being one order of magnitude larger than glass-based materials and almost twice that of silicon [12]. A high thermo-optic coefficient enables to reduce the electrical power consumption of thermal actuators employed in optical waveguides to counteract fabrication tolerances and to tune reconfigurable architectures. Furthermore, SiOC exhibits low loss at telecom wavelengths and can be integrated in most photonic platforms [12].

SiOC thin films can be deposited by using different deposition techniques [13-17]. Chemical vapor deposition (CVD) and sol-gel pyrolysis were used to achieve SiOC films with different compositions. Gallis et al. [5,17] employed thermal CVD to prepare Si-rich through stoichiometric to Crich hydrogenated $\mathrm{SiOC}$ thin films on $\mathrm{Si}$ substrates and demonstrated strong photoluminescence in the visible and near-infrared wavelength ranges. Karakuscu and Guider [6] observed white luminescence from sol-gel derived SiOC thin films produced from triethoxysilane and methyldiethoxysilane precursors. The reported characterizations of the various SiOC films indicate large differences in composition, absorption and refractive index as a function of carbon concentration, postthermal annealing, and deposition techniques. Ryan and Pantano [18,19] reported on the chemical and structural properties of $\mathrm{SiO}_{(2-2 x)} \mathrm{C}_{x}$ films deposited by sputtering a $\mathrm{SiC}$ target, across a continuous range $0 \leq \mathrm{x} \leq 1$ attained by changing the oxygen partial pressure in the sputtering chamber. The synthesized SiOC films were amorphous and appeared to possess no impurities. However, no information of the optical properties of sputter deposited SiOC films were provided. More recently, Mandracci et. al. [20] exploited $\mathrm{SiOC}$ to realize multi-layer structures for the coupling of Bloch Surface Waves, yet not giving information on the absorption coefficient of SiOC in the optical spectral range.

In this work, we present an experimental study on SiOC integrated optical waveguides and we demonstrate the possibility of realizing photonic integrated circuits (PICs) on a SiOC platform. To this aim, reactive RF magnetron sputtering was employed to deposit SiOC thin films over a wide range of material composition, from silica to amorphous silicon carbide (SiC). We anticipate here that, even though sputtering is not the best suited technique to achieve the highest quality films for integrated optical applications, it provides a unique flexibility on material preparation, allowing fast and cheap investigation of the optical properties of the deposited films versus composition. 
TABLE 1: PARAMETERS OF SPUTTERING PROCESS AND SIOC PROPERTIES

\begin{tabular}{|c|c|c|c|c|c|c|c|}
\hline \multicolumn{2}{|c|}{ Sputter Parameter Space of SiOC Films } \\
\hline Sample ID & $\begin{array}{c}\text { RF Power } \\
{[\mathrm{W}]}\end{array}$ & $\begin{array}{c}\text { Ar flow } \\
{[\mathrm{sccm}]}\end{array}$ & $\begin{array}{c}\mathrm{O}_{2} \text { flow } \\
{[\mathrm{sccm}]}\end{array}$ & $\begin{array}{c}\text { Deposition rate } \\
{[\mathrm{nm} / \mathrm{min}]}\end{array}$ & $\begin{array}{c}\mathrm{n} \\
(1550 \mathrm{~nm}\end{array}$ & Morphology & Phase \\
\hline S1 & 50 & 60 & 1.5 & 5.3 & 1.40 & Columnar & Amorp. \\
\hline S2 & 150 & 60 & 1.5 & 30.4 & 1.46 & Columnar & Amorp. \\
\hline S3 & 200 & 60 & 1.5 & 42.7 & 1.48 & Columnar & Amorp. \\
\hline S4 & 300 & 60 & 1.5 & 100 & 1.60 & Columnar & Amorp. \\
\hline S5 & 350 & 60 & 1.5 & 126.7 & 1.71 & Columnar & Amorp. \\
\hline S6 & 450 & 60 & 1.5 & 146 & 1.86 & Columnar & Amorp. \\
\hline S7 & 350 & 10 & 1.5 & 30 & 1.50 & Compact & Amorp. \\
\hline S8 & 350 & 10 & 0.75 & 25 & 2.2 & Compact & Amorp. \\
\hline
\end{tabular}

We identified the sputtering process parameters suitable to produce SiOC films with a different composition (i.e. carbonoxygen ratio), providing refractive index tuning from 1.40 to almost 3.0 in the wavelength range around $1550 \mathrm{~nm}$; this wide tuneability enables to meet most of the requirements in the design of PICs, from low index contrast to highly confined waveguides for high-integration-scale applications.

The paper is structured as follows. In Sec. II we describe the sputtering technique employed for the deposition of the SiOC films, pointing out the most relevant process parameters and their impact on the structural and morphological properties of the deposited films. Section III illustrates the optical properties of the SiOC films, focusing on the tuneability of the refractive index and on its dependence on the material composition. Several examples of waveguide structures with different refractive index contrast are discussed in Sec. IV, while in Sec. V an integrated Mach-Zehnder interferometer (MZI) based on multimode interference (MMI) coupler is presented as a first example of PIC in SiOC technology. A concluding section summarizes the main achievements of this work.

\section{DEPOSITION AND MORPHOLOGY}

Thin films of SiOC were deposited by reactive radio frequency (RF) magnetron sputtering (Leybold LH Z400) from a 3-inch diameter $\mathrm{SiC}$ target (99.9\% purity) using argon and oxygen as plasma and reactive gases, respectively. Depositions were performed at room temperature on p-type silicon (100) and thermally oxidized boron doped p-type $\mathrm{Si}$ (100) substrates. The Ar flow rate was optimized to achieve the best tradeoff between quality of the films, in terms of morphology and deposition rate; the $\mathrm{O}_{2}$ flow is instead responsible for material composition and hence it determines the refractive index and the energy gap of the material. RF power between $50 \mathrm{~W}$ and $450 \mathrm{~W}$ was used, a higher RF power leading to higher deposition rate and higher refractive index. Bare Si substrates were used to optimize the sputter process and measure SiOC film properties while thermally oxidized $\mathrm{Si}$ substrates, with $6 \mu \mathrm{m}$ thick silica layer, were used to fabricate photonic waveguides and PICs.

The multi-dimensional space of parameters of the sputtering process and the impact on the optical properties of the deposited SiOC films were analyzed by depositing several samples, as listed in Table 1. In order to cover the composition range from $\mathrm{SiC}$-like to silica-like compounds, in some samples (samples S1-S6) the RF power was increased while keeping the oxygen flux at $1.5 \mathrm{sccm}$ and the Ar flux at $60 \mathrm{sccm}$; in other samples (S7-S8) the $\mathrm{O}_{2}$ flux was varied between 0.75 sccm and $1.5 \mathrm{sccm}$ while keeping the Ar flux at $10 \mathrm{sccm}$ and $350 \mathrm{~W}$ RF power. Samples deposited at larger Ar flux and with RF power between 50 and $450 \mathrm{~W}$ showed rough, porous and columnar structure with increase in the deposition rate and refractive index ( $n$, measured at a wavelength of $1550 \mathrm{~nm}$ ) from $5.3 \mathrm{~nm} / \mathrm{min}$ to $127 \mathrm{~nm} / \mathrm{min}$ and 1.40 to 1.86 , respectively. Reducing the Ar flux from $60 \mathrm{sccm}$ to $10 \mathrm{sccm}$ provided trade-off between porous structure and the deposition rate. Sample S7 exhibited compact structure and smooth surface and showed higher refractive index value compared to $\mathrm{S} 2$ that has a similar deposition rate. Further reducing $\mathrm{O}_{2}$ flow, SiOC sample S8 with an increased refractive index of 2.2 similar to $\mathrm{Si}_{3} \mathrm{~N}_{4}$ is obtained. At $1550 \mathrm{~nm}$ all the films of Tab. 1 exhibit a loss lower than the accuracy of the ellipsometric technique used for the optical characterization (see Sec. III).

All the samples exhibited amorphous microstructure irrespective of columnar or compact morphology. The adhesion between sputtered SiOC film and $\mathrm{Si}$ or thermally oxidized Si substrates was excellent showing no pin holes or delamination during ultrasonic bath for 30 minutes and successive processes. The residual stress in SiOC films was measured with profilometer following Stoney equation [21,22]. The SiOC film with refractive index of about 2.2 showed low residual compressive stress of around $200 \mathrm{MPa}$, a value reported also in other publications [23].

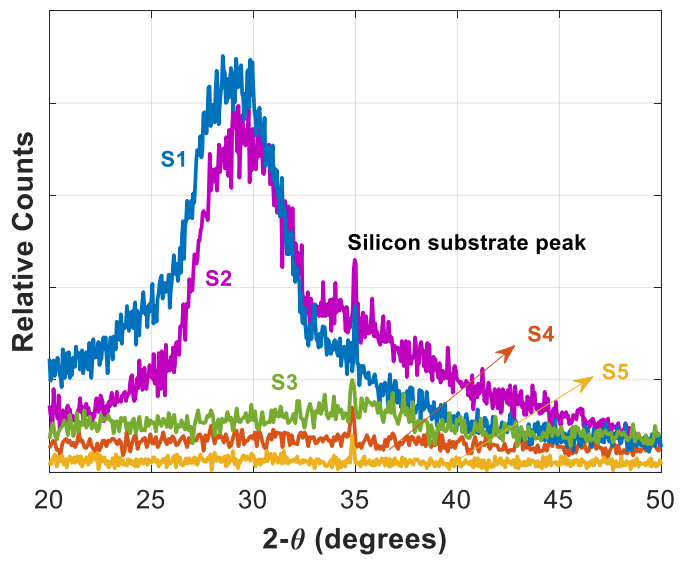

Figure 1: XRD spectra measured over $2 \theta$ Range from 20 to 50 degrees confirms amorphous phase of SiOC films as no peak is detected except $\mathrm{Si}$ at 35 degrees 
The phase structure of the SiOC films deposited at RF power levels between $50 \mathrm{~W}$ and $450 \mathrm{~W}(60 \mathrm{sccm}$ Ar, columnar structure) was investigated with X-ray diffraction (XRD). Figure 1 shows the XRD spectra of SiOC samples (S1 to S5) measured at a grazing angle of incidence $2 \theta$ from 20 to 50 degrees. The measured spectra show no sharp peaks which implies that SiOC films possess an amorphous structure. The sharp peak at $35^{\circ}$ corresponds to Si substrate and the broad peaks in S1 and S2 samples show the presence of silica substructure in $\mathrm{SiOC}$ as these samples have more oxygen content.

Cross-sectional images of the deposited SiOC films were taken with a scanning electron microscope (SEM) to evaluate the film compactness and adhesion. To quantify the surface roughness, atomic force microscopy (AFM) was performed scanning the surface of the SiOC films over an area of 100 $\mu \mathrm{m}^{2}$ in tapping mode. Figure 2(a) shows the SEM image of a $400 \mathrm{~nm}$ thick SiOC film deposited at $60 \mathrm{sccm}$ Ar flow rate and $\mathrm{RF}$ power of $350 \mathrm{~W}$ (deposition rate $130 \mathrm{~nm} / \mathrm{min}$ ). The material exhibits a porous structure with vertical pillars on the scale of tens of nanometers. Such columnar structure was already observed in previous publications $[18,19,23]$, showing that sputtered SiOC films deposited at high Ar flow has a pronounced porosity.
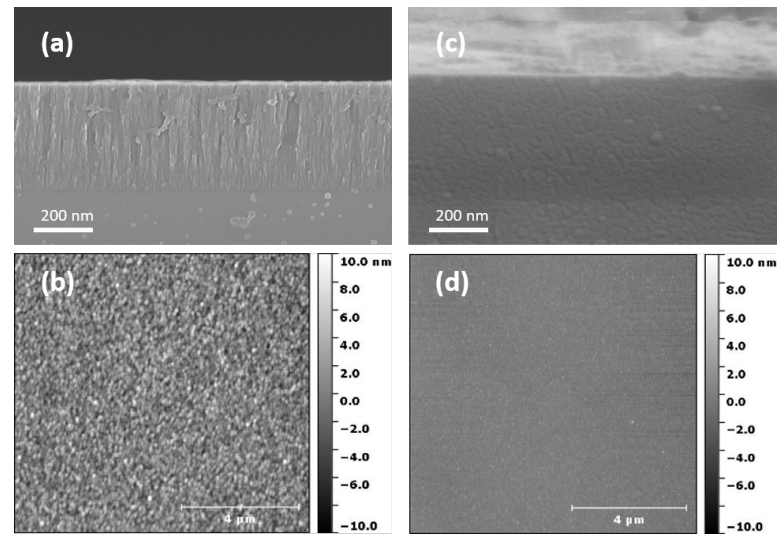

Figure 2: SEM and AFM images of (a)-(b) columnar and (c)(d) compact SiOC films sharing a same thickness of $400 \mathrm{~nm}$.

To achieve a more compact structure, the Ar flow was reduced down to $10 \mathrm{sccm}$, this leading to a reduction of the deposition pressure in the chamber from $8 \times 10^{-2}$ mbar to $3 \times 10^{-2}$ mbar. In this case, the sputtered atoms experience less collisions with other atoms and energetic ions reflected from target surface and arrive more smoothly at the substrate surface $[24,25]$, thus resulting in a reduced deposition rate. As shown in Fig. 2(c), in a 400-nm thick SiOC film deposited at $10 \mathrm{sccm}$ Ar flow (deposition rate $30 \mathrm{~nm} / \mathrm{min}$ ) no columnar features can be seen. The higher compactness of the film results also in a smoother surface. From the 2D AFM acquisitions of Fig. 2 (b) and (d), a surface roughness reduction from $1 \mathrm{~nm}$ to less than $0.25 \mathrm{~nm}(\mathrm{rms})$ is observed, the latter being comparable with the roughness of the silica substrate. If porous films can be effectively exploited in applications such as sensing [26], photonic waveguides require indeed compact and uniform films to have low propagation loss.
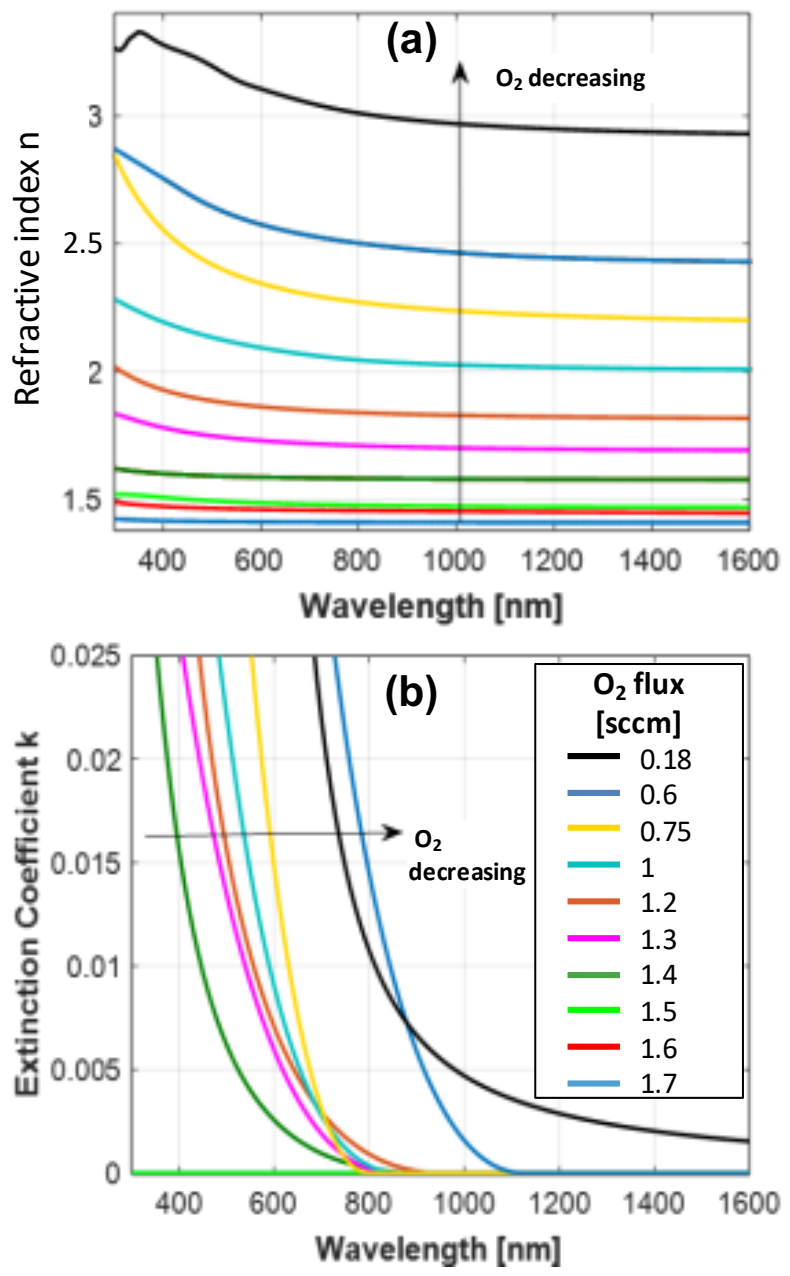

Figure 3: (a) refractive index and (b) extinction coefficient spectra of SiOC films deposited under varying $\mathrm{O}_{2}$ flow and setting $R F$ power at $350 \mathrm{~W}$ and Ar flow at $10 \mathrm{sccm}$ as obtained from ellipsometric measurements. The quantitative relation between the increase of the refractive index and the $\mathrm{O}_{2}$ gas flow is reported in Fig. 4(a).

\section{REFRACTIVE INDEX TUNING}

The material composition and hence the optical properties of SiOC films strictly depend on the $\mathrm{O}_{2} / \mathrm{Ar}$ gas flow ratio and the RF sputtering power. In this section this relation is studied in details for both columnar and compact films. Several films were deposited by varying the RF power from $50 \mathrm{~W}$ to $450 \mathrm{~W}$ and the $\mathrm{O}_{2}$ flow from $0 \mathrm{sccm}$ to $3 \mathrm{sccm}$, the Argon flux being kept at either $60 \mathrm{sccm}$ or $10 \mathrm{sccm}$. The refractive index $(n)$ and extinction coefficient $(k)$ of each film were measured by spectroscopic ellipsometry in the visible and near-IR range $(300 \mathrm{~nm}-1600 \mathrm{~nm})$. Measurements were performed at three angles of incidence $65^{\circ}, 70^{\circ}$ and $75^{\circ}$ and were fitted using a layer stack model including surface roughness for the SiOC film, $\mathrm{SiO}_{2}$ layer, and $\mathrm{Si}$ substrate [11]. The $\mathrm{SiOC}$ film was modelled using Cody-Lorentz model [27,28], providing physical based values of $n$ and $k$ reported in Fig. 3(a) and (b), 
respectively. Results show that as the $\mathrm{O}_{2}$ gas flow rate decreases with respect to Ar flow set at $10 \mathrm{sccm}$ (RF power kept at $350 \mathrm{~W}$ ), the refractive index increases across the entire visible and near-IR wavelength range.
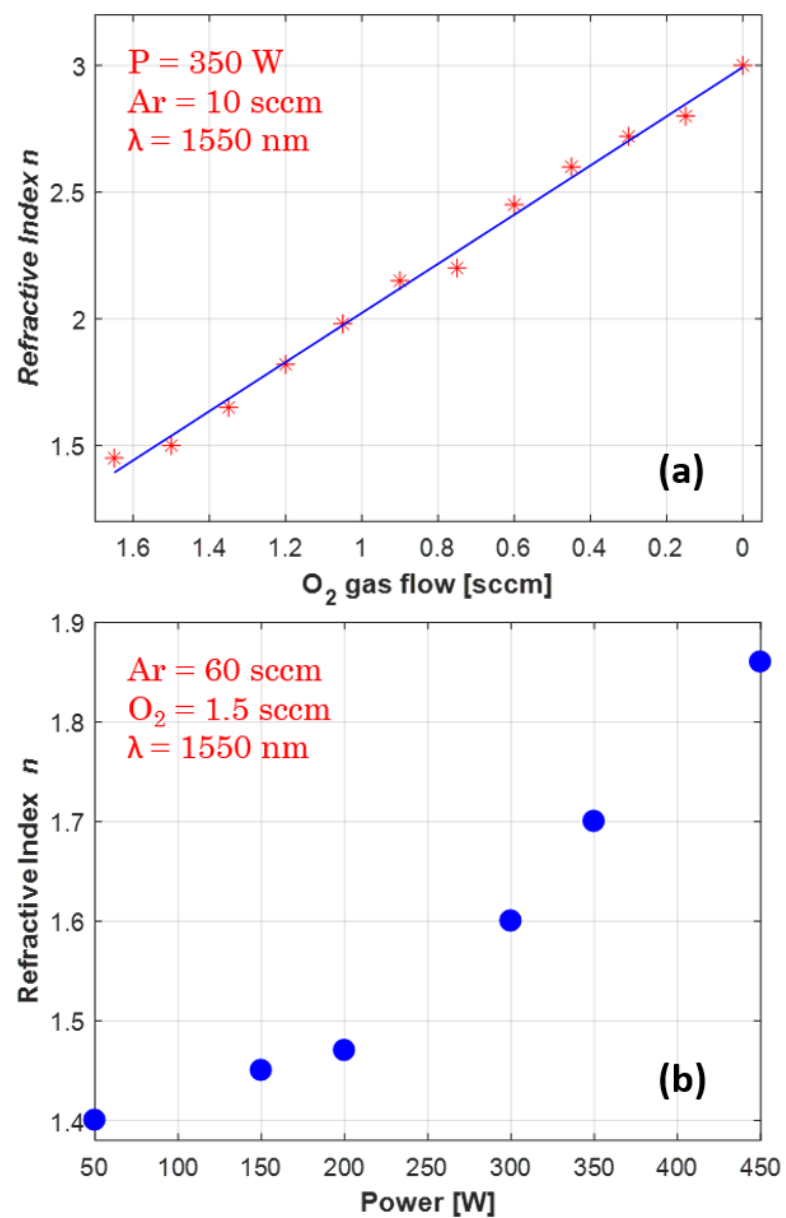

Figure 4: (a) Increase in refractive index of SiOC films as a function of $\mathrm{O}_{2}$ gas flow when Ar gas flow and $\mathrm{RF}$ power are constant at $10 \mathrm{sccm}$ and $350 \mathrm{~W}$, (b) increase in refractive index of SiOC films as a function of RF power $(50$ to $450 \mathrm{~W})$ when $\mathrm{Ar}$ and $\mathrm{O}_{2}$ gas flow are constant at $60 \mathrm{sccm}$ and 1.5 sccm

The quantitative relation between the refractive index measured at a wavelength of $1550 \mathrm{~nm}$ and the $\mathrm{O}_{2}$ gas flow is reported in Fig. 4(a). Further, when the Ar flux is set at 60 $\mathrm{sccm}$, the refractive index increases from 1.40 to 1.86 as a function of RF power in the power range from $50 \mathrm{~W}$ to 450 $\mathrm{W}$, as given in Fig. 4(b). This effect is associated with a change in the composition of the material, that is in relative amount of silicon $(\mathrm{Si})$, oxygen $(\mathrm{O})$ and carbon $(\mathrm{C})$ in the film.

X-ray photoelectron spectroscopy (XPS) measurements reported in Fig. 5(a) show the composition of the SiOC films versus the refractive index. Data are reported for columnar SIOC films with a refractive index comprised between 1.41 and 1.86, and for a compact film with a refractive index of 2.2. $\mathrm{C} 1 \mathrm{~s}, \mathrm{O} 1 \mathrm{~s}$ and $\mathrm{Si} 2 \mathrm{p}$ core levels were excited by standard $\mathrm{Al} \mathrm{K \alpha}$ $\mathrm{X}$-ray source and recorded using a a Hemispherical Energy Analyzer (HEA) Phoibos 150 (SPECS $^{\mathrm{TM}}$ ), yielding an acceptance angle of $\pm 2.5^{\circ}$, a field view of $\sim 1.4 \mathrm{~mm}^{2}$ and an energy resolution of $0.95 \mathrm{eV}$. In the columnar films, the increase of refractive index can be related to the increase of carbon content (orange dots), which replaces oxygen atoms (blue squares) in the SiOC compound when the RF power is increased, while the amount of $\mathrm{Si}$ (gray stars) is almost unchanged. Such increase of the $\mathrm{C}$ content induced by the decrease of the $\mathrm{O}_{2}$ gas flow is indeed in line with observations reported in the literature [17,18]. In contrast, the higher refractive index of the compact film is associated to an increase of the Si ratio. Fitting the XPS spectra by different components ( $\mathrm{SiO}$ and $\mathrm{CO}$ for $\mathrm{O} 1 \mathrm{~s} ; \mathrm{SiC}, \mathrm{SiO}$ and $\mathrm{SiSi}$ for $\mathrm{Si} 2 \mathrm{p}$; $\mathrm{SiC}, \mathrm{CC}$ and $\mathrm{CO}$ for $\mathrm{C} 1 \mathrm{~s}$ ) allows to identify $\mathrm{Si}-\mathrm{C}$ and $\mathrm{C}-\mathrm{O}$ as the predominant chemical bonds in the SiOC samples [29]. The accuracy in determining the material composition is \pm $2.5 \%$. XPS also reveals no trace of impurity in the deposited films, within our accuracy of about $3 \%$. Further comparison between this work and [18] is given in Fig. 5(b) that are in good agreement to each other.
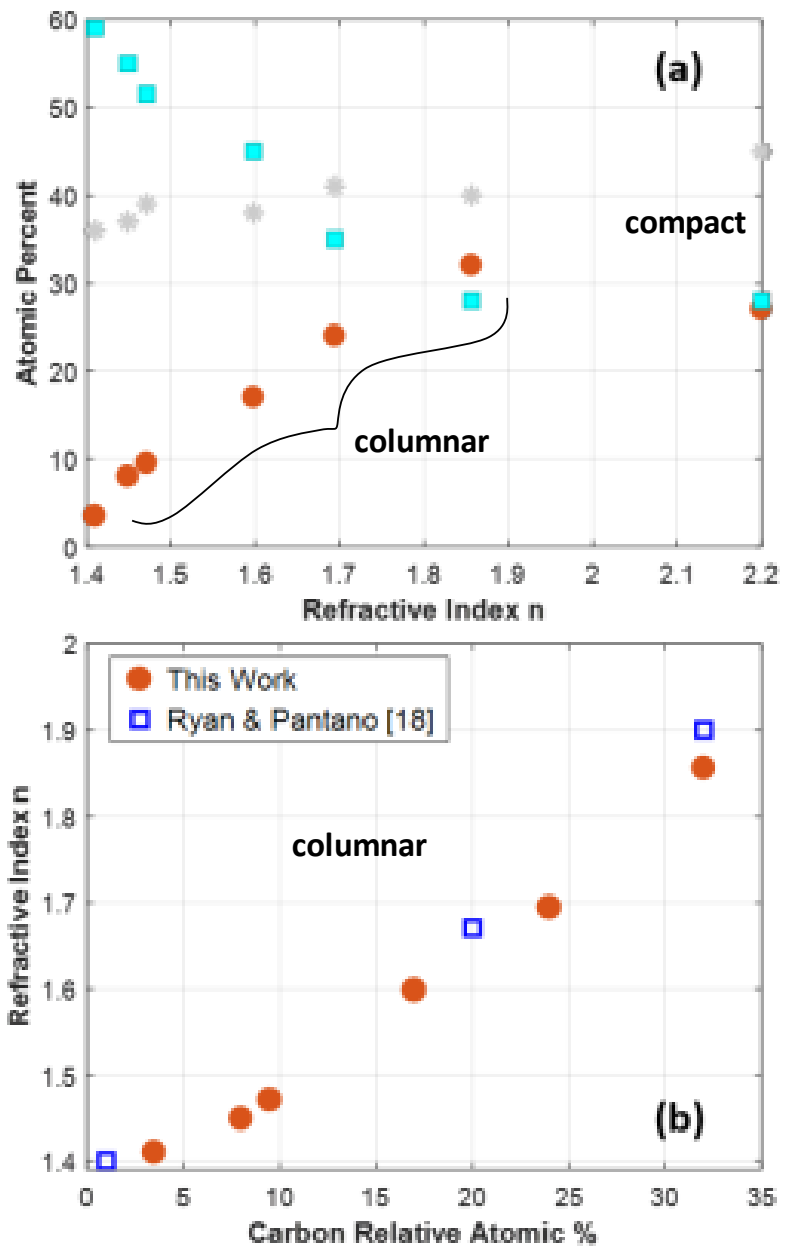

Figure 5: (a) XPS elemental analysis plotted as a function of refractive index of SiOC films, (b) comparison between carbon content $v / s$ refractive index of SiOC films deposited in this work and [18] 
TABLE 2: PROPERTIES AND PARAMETERS OF FABRICATED SiOC WAVEGUIDES

\begin{tabular}{|c|c|c|c|c|c|}
\hline Core Index & Morphology & $\begin{array}{c}\text { Index Contrast } \\
\boldsymbol{\Delta} \mathbf{n}\end{array}$ & $\begin{array}{c}\text { Dimensions } \\
{[\boldsymbol{\mu} \mathbf{m}]}\end{array}$ & $\begin{array}{c}\text { Propagation Loss } \\
{[\mathbf{d B} / \mathbf{c m}]}\end{array}$ & Group index \\
\hline $1.578[11]$ & Columnar & $8 \%$ & $4.4 \times 0.4$ & $4 \pm 0.5$ & 1.495 \\
\hline 1.819 & Columnar & $19 \%$ & $1.8 \times 0.4$ & $8 \pm 0.6$ & 1.722 \\
\hline 2.20 & Compact & $28 \%$ & $1.3 \times 0.175$ & $2 \pm 0.5$ & 1.995 \\
\hline
\end{tabular}

Figure 3(a) shows that by increasing the $\mathrm{C}$ content the refractive index of SiOC can be tailored from less than that of silica, that is $n=1.40$ at a wavelength of $1550 \mathrm{~nm}$, to that of a$\mathrm{SiC}$, that is about 2.93 . The absolute accuracy of the measured refractive index is in the order of $\pm 10^{-2}$, as confirmed by measurements carried out on the same samples by using prism coupling technique at $633 \mathrm{~nm}$ and $1550 \mathrm{~nm}$. The low refractive index of 1.40 of SiOC is attributed to high porosity of the material structure, which is exploited as interlayer dielectrics in microelectronics $[4,30]$. In the films with compact structure, no evidence of material anisotropy was observed in the ellipsometric measurements as reported in Fig. 3(a), while SiOC films with columnar structures exhibited a refractive indices difference as large as $10^{-2}$ between in-plane and off-plane axes [11].

The change of the refractive index is associated with a modification of the energy gap of the material $E_{\mathrm{g}}$, which moves to lower energy when the $\mathrm{C}$ content increases, as shown in Fig. 3(b). The value of the energy bandgap was extracted from the absorption spectra by using Tauc relation $(\alpha h \omega)^{2}=h \omega-E_{\mathrm{g}}$, where $\alpha$ is absorption coefficient and $h \omega$ is photon energy [31]. As the refractive index increases from 1.6 to almost $3.0, E_{\mathrm{g}}$ decreases from $3.8 \mathrm{eV}$ to $1.81 \mathrm{eV}$ and is responsible for the increase of the extinction coefficient at longer wavelengths. However, at $1550 \mathrm{~nm} k$ is below the accuracy of the ellipsometric measurement $\left(10^{-3}\right)$ as long as the refractive index is lower than 2.5. Below $800 \mathrm{~nm}$, the photon energy exceeds the energy gap of the material (especially for higher refractive index SiOC) and the deposited films exhibit higher losses.

A remarkable advantage of $\mathrm{SiOC}$ is the extended achievable range of refractive index, much wider than that of silicon oxynitride ( $\mathrm{SiON}$ ) [32-35] and comparable to silicon-rich nitride (SRN) [28]. Moreover, the absence of any absorption peak around the $1500 \mathrm{~nm}$ wavelength range makes this material an excellent candidate for applications in the telecom wavelength range, especially with respect to $\mathrm{SiON}$.

\section{PHOTONIC WAVEGUIDES FABRICATION}

The tuneability of the refractive index of SiOC films has been investigated to realize photonic waveguides with different refractive index contrast. The index contrast is defined as $\Delta n$ $=\left(n-n_{c l}\right) / n$, where $n$ is the refractive index of the SiOC core material and $n_{c l}$ is the refractive index of the cladding, which in all the examples reported in this work is made of $\mathrm{SiO}_{2}\left(n_{\mathrm{cl}}=\right.$ 1.444). Namely, waveguides with an index contrast $\Delta n=8.2 \%$ $(n=1.578), \Delta n=19 \%(n=1.819)$ and $\Delta n=28 \%(n=2.20)$ were fabricated. In the first two cases, SiOC films deposited with $60 \mathrm{sscm}$ Ar flow rate and exhibiting a columnar structure were employed, while the third one, which was deposited with $10 \mathrm{sscm}$ Ar flow rate, has a compact SiOC film.
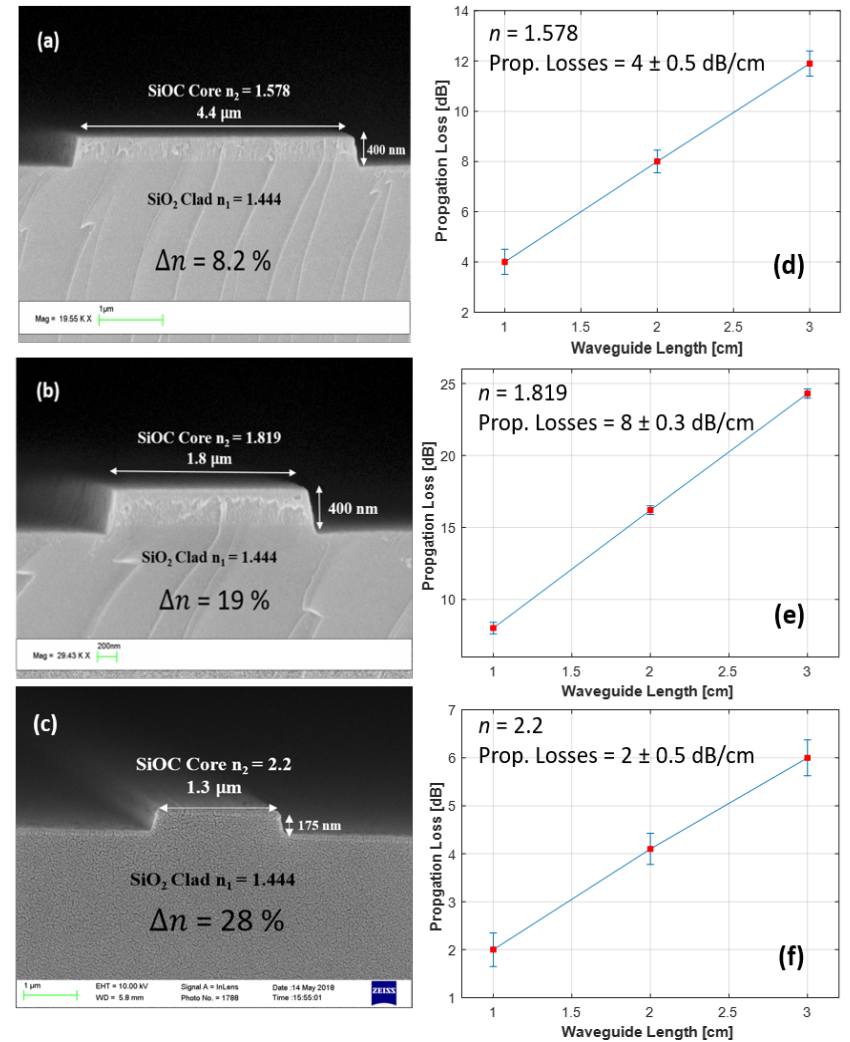

Figure 6: (a)-(c) SEM images and (d)-(f) propagation loss plots of fabricated SiOC waveguides with refractive indices of $n=1.578, n=1.819$ and $n=2.20$, respectively

To define the waveguide structures, AZ 5214 E photoresist was spin coated on the SiOC layer and soft baked on a hot plate at $110{ }^{\circ} \mathrm{C}$; the photoresist was patterned by direct-laser writing photolithography $(365 \mathrm{~nm})$ followed by inductively coupled plasma reactive ion etching (ICP-RIE) in $\mathrm{CHF}_{3}(100$ sccm) and $\mathrm{O}_{2}(5 \mathrm{sccm})$. The etch rate of the SiOC films is strongly dependent on the refractive index, being $20 \mathrm{~nm} / \mathrm{min}$ for $n=1.578$ and $9 \mathrm{~nm} / \mathrm{min}$ for $n=2.20$. The fabricated channel waveguides show vertical sidewall profile with angle of about $85^{\circ}$. A common core thickness of $400 \mathrm{~nm}$ is used for $\Delta n=8.2 \%(n=1.578)$ and $\Delta n=19 \%(n=1.819)$ while 175 $\mathrm{nm}$ for $\Delta n=28 \%(n=2.20) \mathrm{SiOC}$ waveguides. The thickness and width were adjusted to operate in single mode regime. A strip waveguide cross section has been used in order to reduce the etching depth and consequently radiation losses induced by sidewall backscatter. Finally, a $4 \mu$ m-thick $\mathrm{SiO}_{2}$ upper cladding layer was deposited by plasma enhanced chemical vapor deposition (PECVD). Waveguides characteristics and 
other data are reported in Table 2. SEM photographs of the fabricated waveguide before the deposition of the silica upper cladding are shown in Figs. 6 a)-c).

The propagation loss of the fabricated waveguides was measured by standard cut-back technique. Lensed optical fibers were used to couple the light into the waveguide from a tunable laser source operating in the $1500 \mathrm{~nm}-1600 \mathrm{~nm}$ wavelength range and the output light was measured by an optical spectrum analyzer. The polarization state of the light at the input facet of the waveguide was selected with a polarization controller guaranteeing at least $-30 \mathrm{~dB}$ crosstalk between transverse electric (TE) and transverse magnetic (TM) polarizations.
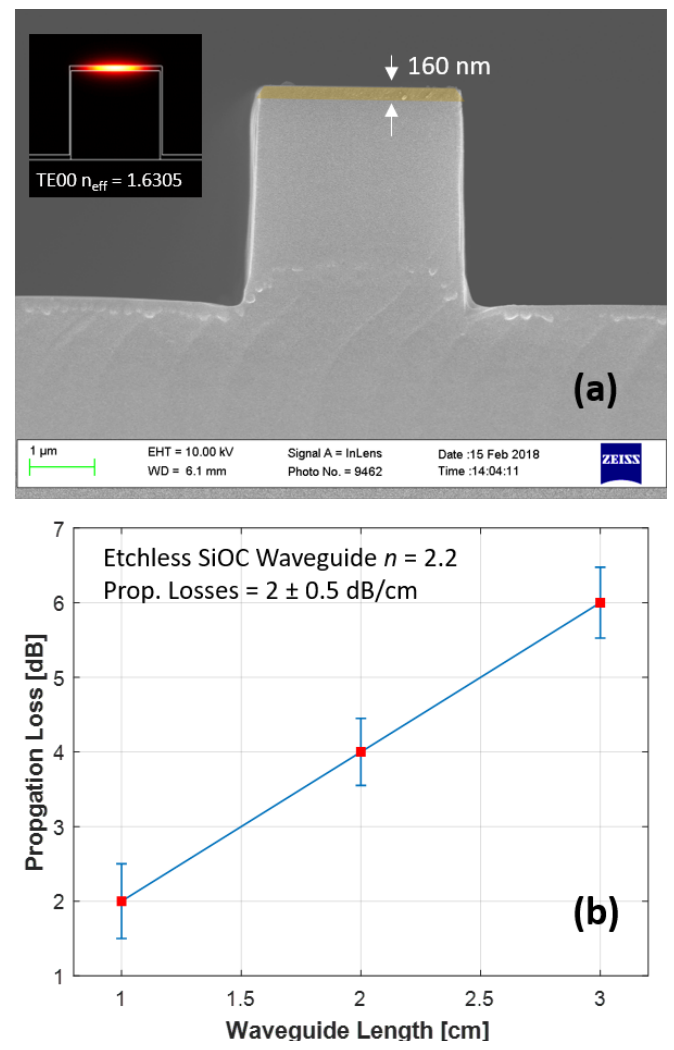

Figure 7: (a) SEM image of an etchless SiOC waveguide ( $n=$ 2.2) with a thickness $=160 \mathrm{~nm}$ and (b) propagation loss plotted against waveguide length

Figures 6 (d)-(f) show the transmission of the three waveguides (a)-(c) versus the waveguide length. For the waveguides with core refractive index $n=1.578$ and $n=1.819$ a propagation loss of about $4 \mathrm{~dB} / \mathrm{cm}$ [11] and $8 \mathrm{~dB} / \mathrm{cm}$ have been measured. In contrast, the SiOC waveguide with higher index $n=2.20$ exhibits propagation losses of only $2 \mathrm{~dB} / \mathrm{cm}$. We attribute such loss improvements to the reduction of the volume scattering, which in the lower index waveguides 6 (a) and 6 (b) is induced by the columnar structure of SiOC film that is the main origin of loss. Absorption loss is likely to give a marginal contribution because $\mathrm{SiOC}$ absorption in the nearIR range is expected to increase with the refractive index as a consequence of the bandgap energy lowering [see Fig. 3(b)].
Likewise, scattering induced by sidewall roughness has a significant contribution. This analysis confirm that SiOC is an excellent candidate for integrated optical applications and a lower attenuation is expected with a careful reduction of the sidewall roughness, possibly by using PECVD deposition of the film [20] and even annealing, in addition to an optimization of the RIE process, all technological improvements left to future studies. Measured waveguide attenuations of TE and TM modes are comparable within the uncertainty of the characterization technique.

The group index of the waveguides was measured too, by using an optical frequency domain reflectometry technique (OFDR) [36-38] and reported in Table 2. Since SiOC films with $n=1.578$ and $n=1.819$ show columnar structure, the waveguides exhibited group birefringence on the order of $10^{-3}$ that further confirmed anisotropy measured with ellipsometry. The details can be found in our previous published work [11].

To better understand the origin of the propagation loss we fabricated etch-less waveguides with the geometry shown in Fig 7(a). A 160-nm thick layer of $\mathrm{SiOC}(n=2.20)$ is deposited on top of a patterned $\mathrm{SiO}_{2}$ substrate ridge $3.1 \mu \mathrm{m}$ wide and 3.3 $\mu \mathrm{m}$ high. At the vertical sidewalls of the $\mathrm{SiO}_{2}$ substrate, the thickness of the SiOC film is about $60 \mathrm{~nm}$ and the optical mode is almost completely confined in the top horizontal SiOC layer as shown in the inset of Fig. 7 (a). The SiOC film has been highlighted in false color. A propagation loss of $2 \pm$ $0.5 \mathrm{~dB} / \mathrm{cm}$ was measured [see Fig. 7(b)], which is comparable to that of the etched waveguide of Fig. 6(c). Since we observe the same propagation loss in etched and etchless waveguide, we can exclude light scattering due to sidewall roughness as the main source of the loss. We estimate that loss is mainly due to volume scattering because of residual material inhomogeneity related to the sputtering process, even though the material looks compact.

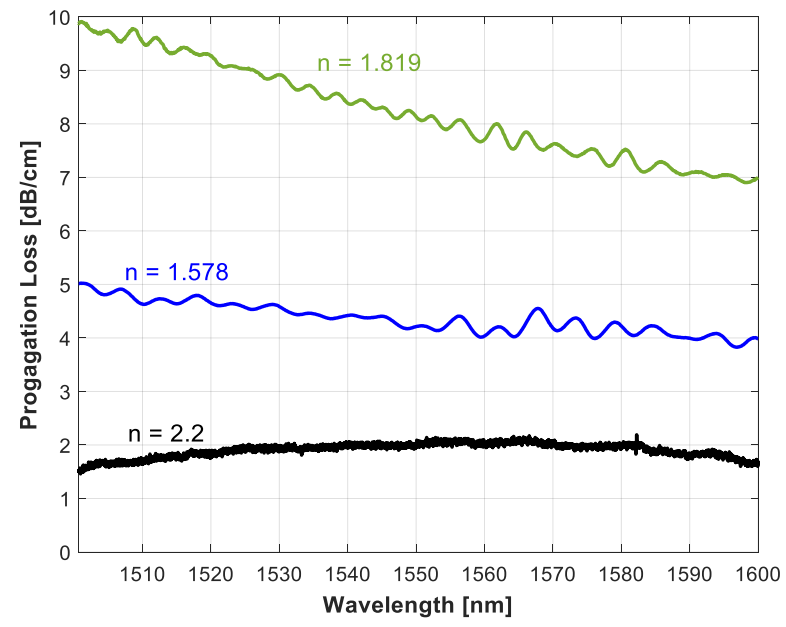

Figure 8: Transmission spectrum of SiOC waveguides with core refractive index $n=1.578, n=1.819$ and $n=2.20$

Figure 8 shows the transmission spectra of the three waveguides of Fig. 6 over a wavelength window from 1500 to $1600 \mathrm{~nm}$. Noteworthy, not only the SiOC waveguide with compact morphology $(n=2.20)$ shows the lowest propagation 
loss, but the transmission spectrum is also maximally flat. In contrast, in the waveguides with a lower SiOC refractive index, where the propagation loss is higher and volume scattering is more pronounced, some spectral ripples appears in the loss profile. Furthermore, the propagation loss increases at shorter wavelengths where the contribution of the scattering induced by the columnar structure becomes more relevant. The flat transmission of the high index $\mathrm{SiOC}$ waveguide makes it a promising candidate for broadband applications.

\section{PHOTONIC DEVICES}

To further demonstrate the suitability of the SiOC technology to realize photonic integrated circuits we designed, fabricated and tested Mach-Zehnder interferometers (MZI). The SiOC channel waveguide has a core refractive index of 2.20 , thickness of $175 \mathrm{~nm}$ and width of $1.3 \mu \mathrm{m}$, in order to operate in single-mode regime. The integrated power splitters were realized by using $2 \times 2$ multimode interference (MMI) couplers, which were designed according to a paired interference approach [39] for 50/50 splitting ratio at the wavelength of $1550 \mathrm{~nm}$. The multimode waveguide of both MMIs has a length $L_{\mathrm{MMI}}=100 \mu \mathrm{m}$ and a width $W_{\mathrm{MMI}}=12 \mu \mathrm{m}$, and the position of access waveguides is located in $\pm \mathrm{W}_{\mathrm{MMI}} / 6$ so as to provide a center-to-center gap of $4 \mu \mathrm{m}$, which is large enough to avoid an evanescent coupling between them. An SEM image of the MMI coupler is shown in Fig. 9(a) with inset showing access waveguides and Fig. 9(b) shows the optical micrograph of the fabricated MZI in SiOC.

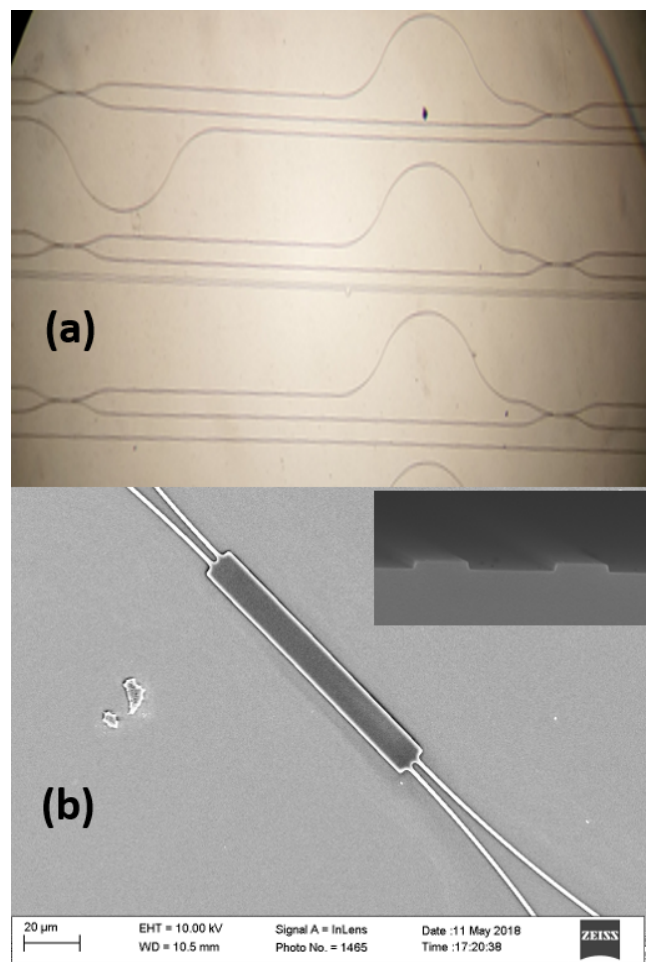

Figure 9: (a) SEM image of MMI with $L_{M M I}=100 \mu \mathrm{m}$ and $W_{M M I}=12 \mu \mathrm{m}$, inset shows access waveguides. (b) Optical micrograph of the MMI based MZI
The MZI was designed to have an unbalance of $243.3 \mu \mathrm{m}$ using a waveguide bending radius of $474 \mu \mathrm{m}$ to prevent additional bending losses. This unbalance corresponds to an FSR $=4.95 \mathrm{~nm}$, as verified experimentally in Fig. 10. The end-to-end length of the MZI is about $5 \mathrm{~mm}$.

Figure 10 shows the transmission spectrum measured at bar and cross output ports of the MZI device. The peak intensity at the cross and bar outputs differ by a fraction of $\mathrm{dB}$, confirming the low attenuation of the waveguides. As a comparison, the solid black line shows the transmission of a straight waveguide with the same length of the MZI. The excess loss of the MZI, amounting to less than $1 \mathrm{~dB}$ over the entire wavelength range, is mainly caused by two MMI splitters, which is estimated to be of about $0.4 \mathrm{~dB}$ each. The extinction ratio ( $>15 \mathrm{~dB}$ at the cross port, $>10 \mathrm{~dB}$ at the bar port) is limited by some unequal splitting in the MMIs (about 10\%), which is due to fabrication tolerances.

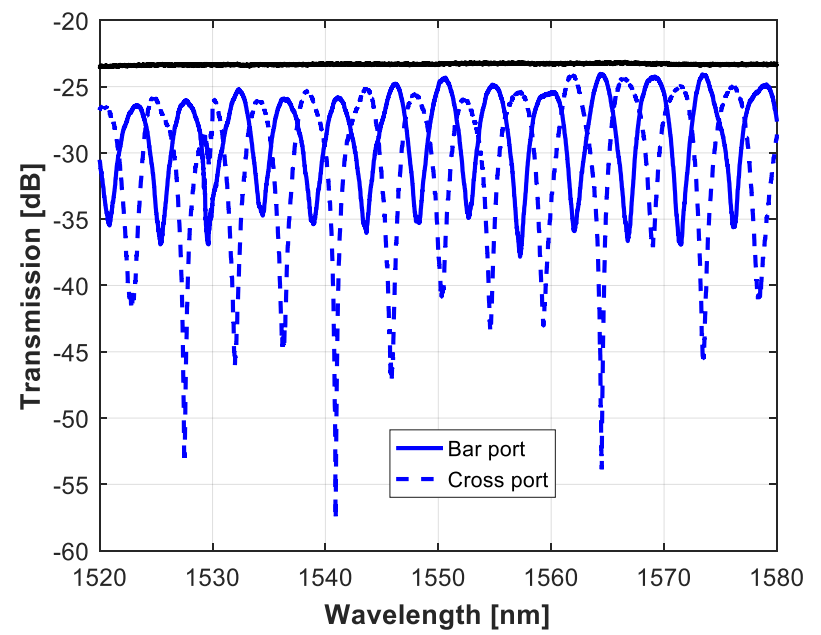

Figure 10: Transmission spectrum measured at Bar and Cross ports of SiOC MZI, showing cross talk less than -10dB. The Transmission has been normalized to that of a straight waveguide with identical width placed on the same chip

\section{CONCLUSION}

We presented an experimental study on SiOC thin films and optical waveguides, demonstrating the possibility of realizing PICs on a SiOC platform.

The structural, morphological and optical properties of SiOC thin films deposited by reactive RF-magnetron sputtering were deeply investigated, showing low level of impurities, low stress, good compactness and homogeneity. By changing the process parameters during the sputtering process ( $\mathrm{Ar}$ and $\mathrm{O}_{2}$ flux, RF power), the carbon-to-oxygen ratio in the deposited film can be finely controlled, this composition change being associated with a wide tuneability of the SiOC refractive index. We achieved SiOC films with a refractive index ranging from less than that of silica (about 1.40) to almost that of amorphous $\mathrm{SiC}$ (almost 3.0), this tuning range widely exceeding the one provided by SiON and being comparable to that covered by SRN [28]. Therefore, SiOC enables to cover a tunable refractive index range that is significantly higher than that provided by other low loss photonic platforms, such as $\mathrm{Si}_{3} \mathrm{~N}_{4}(n=1.99)$ [40] and $\mathrm{Ta}_{2} \mathrm{O}_{5}(n=2.05)$ [41]. Our results 
also show that, as long as the refractive index is lower than 2.5 , the material loss is very low in the near-IR range. The absence of absorption peaks around $1500 \mathrm{~nm}$ wavelength makes this material an excellent candidate for telecom applications.

Several waveguide structures were realized and tested to assess their potential for PICs at telecom wavelengths. Single mode waveguides exhibit propagation losses as low as 2 $\mathrm{dB} / \mathrm{cm}$ across the full telecom window from $1500-1600 \mathrm{~nm}$. The propagation loss is mainly caused by volume scattering due to residual material inhomogeneity related to the sputtering process, which also limits the maximum thickness of the film that can be deposited with a compact structure to less than $500 \mathrm{~nm}$. We expect that thicker waveguides with lower loss can be achieved by using a CVD process for the deposition of the SiOC film. As a consequence of the CVD process, some residual hydrogen could be present in the deposited material, which could cause losses from vibrational overtones; however, with respect to $\mathrm{SiON}$, where the absorption peak of the $\mathrm{N}-\mathrm{H}$ bond overtone is located at 1510 $\mathrm{nm}$, in the case of $\mathrm{SiOC}$ the first $\mathrm{C}-\mathrm{H}$ bond overtone is expected to appear above $1650 \mathrm{~nm}$, so well outside the main wavelength range of telecom applications.

We also reported on a first simple example of PIC realized on high refractive index SiOC platform $(n=2.2)$, consisting of a MMI based unbalanced MZI.

The high tuneability of the refractive index, from low index contrast to highly confined waveguides, together with the high thermo-optic coefficient reported in a previous contribution [12], make SiOC a promising alternative to conventional dielectric platforms for the realization of PICs.

\section{ACKNOWLEDGEMENTS}

This work was mainly performed at Polifab (www.polifab.polimi.it), the micro/nano fabrication facility at Politecnico di Milano, Italy.

\section{REFERENCES}

[1] L. David, R. Bhandavat, U. Barrera and G. Singh, "Silicon oxycarbide glass-graphene composite paper electrode for long-cycle lithium-ion batteries," Nat. Commun., vol. 7, no. 10998, Mar. 2016.

[2] V. S. Pradeep, M. Graczyk-Zajac, R. Riedel and G.D. Soraru, "New Insights in to the Lithium Storage Mechanism in Polymer Derived SiOC Anode Materials," Electro. Acta, vol. 119, no. 10, pp. 78-85 Feb. 2014.

[3] G. Alfred, "Plasma enhanced chemical vapor deposited SiCOH dielectrics: from low-k to extreme low-k interconnect materials," J. App. Physics, vol. 93, no. 3, pp. 1785-1790, Feb. 2003

[4] H. J. Kim, Q. Shao and Yoon-Hae Kim, "Characterization of lowdielectric-constant SiOC thin films deposited by PECVD for interlayer dielectrics of multilevel interconnection," Sur. and Coat. Technology, vol. 171, no. 1-3, pp. 39-45, Jul. 2003.

[5] V. Nikas, S. Gallis, M. Huang, A. E. Kaloyeros, A. P. D. Nguyen, A Stesmans, and V. V. Afanas'ev, "The origin of white luminescence from silicon oxycarbide thin films," App. Phys. Letters, vol. 104, no. 061906, pp. 1-3, Feb. 2014.

[6] A. Karakuscu, R. Guider, L. Pavesi and G. D. Sorarù, "White Luminescence from Sol-Gel-Derived SiOC Thin Films," J. Am. Ceram. Society,vol. 92, no. 12, pp. 2969-2974, Nov. 2009.
[7] Y. Ding, H. Shirai, and D. He, "White light emission and electrical properties of silicon oxycarbide-based metal-oxide-semiconductor diode," Th. Sol. Films, vol. 519, no. 8, pp. 2513-2515, Feb. 2011.

[8] G. Bellocchi, G. Franzò, M. Miritello and F. Iacona, "White light emission from Eu-doped SiOC films," App. Phys. Express, vol. 7, no. 012601, pp. 1-3, Dec. 2013.

[9] S. Gallis, M. Huang and A. E. Kaloyeros, "Efficient energy transfer from silicon oxycarbide matrix to $\mathrm{Er}$ ions via indirect excitation mechanisms," App. Phys. Letters, vol. 90, no. 161914, pp.1-3, Apr. 2007.

[10] G. Bellocchi, F. Iacona, M. Miritello, T. Cesca and G. Franzò, "SiOC thin films: an efficient light source and an ideal host matrix for Eu2+ ions," Opt. Express, vol. 21, no. 17, pp. 20280-20290, Aug. 2013.

[11] F. A. Memon, F. Morichetti, and A. Melloni, "Waveguiding light into silicon oxycarbide," App. Sciences, vol. 7, no. 561, pp. 1-11, May 2017.

[12] F. A. Memon, F. Morichetti, and A. Melloni, "High thermo-optic coefficient of silicon oxycarbide photonic waveguides," ACS Photonics, vol. 5, no. 7, pp. 2755-2759, Jun. 2018.

[13] C. G. Pantano, A. K. Singh and H. Zhang, "Silicon Oxycarbide Glasses," J. Sol-Gel Sc. and Technology vol. 14, no. 1, pp. 7-25, Mar. 1999.

[14] S. L. Shevchuk and Yu. P. Maishev, "Silicon oxycarbide thin films deposited from viniltrimethoxysilane ion beams," Th. Sol. Films, vol. 492, no. 1-2, pp. 114-117, Dec. 2005.

[15] A. Walkiewicz-Pietrzykowska, J. P. Espinós, González-Elipe and R. Agustin, "Type of precursor and synthesis of silicon oxycarbide $(\mathrm{SiOxCyH})$ thin films with a surfatron microwave oxygen/argon plasma,” J. Vac. Sc. \& Technology A, vol. 24, no. 4, pp. 988-994, Jul. 2006.

[16] G. D. Sorarù, G. D'Andrea, R. Campostrini, F. Babonneau and G. Mariotto, "Structural Characterization and High-Temperature Behavior of Silicon Oxycarbide Glasses Prepared from Sol-Gel Precursors Containing Si-H Bonds," J. Am. Ceram. Society, vol. 78, no. 2, pp. 379387, Feb. 1995

[17] S. Gallis, V. Nikas, M. Huang, E. Eisenbraun and A. E. Kaloyeros, "Comparative study of the effects of thermal treatment on the optical properties of hydrogenated amorphous silicon-oxycarbide," J. App. Physics, vol. 102, no. 024302, pp. 1-9, Jul. 2007.

[18] J. V. Ryan, and C. G. Pantano, "Synthesis and characterization of inorganic silicon oxycarbide glass thin films by reactive rf-magnetron sputtering," J. Vac. Sc. \& Technology A, vol. 25, no. 1, pp. 153-159, Jan. 2007

[19] J. V. Ryan, P. Colombo, J. A. Howell and C. G. Pantano, "TribologyStructure Relationships in Silicon Oxycarbide Thin Films," Intl. Jour. of App. Cer. Technology, vol. 7, no. 5, pp. 675-686, Apr. 2009.

[20] P. Mandracci, F. Frascella, R. Rizzo, A. Virga, P. Rivolo, E. Descrovi and F. Giorgis, "Optical and structural properties of amorphous siliconnitrides and silicon-oxycarbides: Application of multilayer structures for the coupling of bloch surface waves," Jour. Non-Cryst. Solids, vol. 453, no. 1, pp. 113-117, Dec. 2016.

[21] G. Franceschinis, "Surface Profilometry as a tool to Measure Thin Film Stress, A Practical Approach," Microelectronics Engineering Department, RIT, 2005.

[22] KLA Tencor Profilometer P-15 User's Guide, Chapter \# 14, Mar. 2005.

[23] P. Du, X. Wang, I. K. Lin, X. Zhang, "Effects of composition and thermal annealing on the mechanical properties of silicon oxycarbide films," Sens. Actuators, A, vol. 176, pp. 90-98, Apr. 2012.

[24] A. Spadoni and M. I. Addonizio, "Effect of the RF sputtering power on microstructural, optical and electrical properties of $\mathrm{Al}$ doped $\mathrm{ZnO}$ thin films," Th. Sol. Films, vol. 589, no. 31, pp. 514-520, Aug. 2015.

[25] E. Gagaoudakis, M. Bender, E. Douloufakis, N. Katsarakis, E. Natsakou, V. Cimalla and G. Kiriakidis, "The influence of deposition parameters on room temperature ozone sensing properties of InOx films," Sen. and Actuators B: Chemical, vol. 80, no. 2, pp.155-161, Nov. 2001.

[26] A. Karakuscu, A. Ponzoni, P. R. Aravind, G. Sberveglieri and G. D. Soraru, "Gas sensing behavior of mesoporous SiOC glasses," Jour. Amer. Cer. Society, vol. 96, no. 8, pp.2366-2369, Jul. 2013.

[27] A. S. Ferlauto, G. M. Ferreira, J. M. Pearce, C. R. Wronski, R. W. Collins, X. Deng and G. Ganguly, "Analytical model for the optical functions of amorphous semiconductors from the near-infrared to ultraviolet: Applications in thin film photovoltaics," Jour. App. Phys, vol. 92, no. 5, pp. 2424-2436, Sep. 2002.

[28] D.K. Ng, Q. Wang, T. Wang, S. K. Ng, Y. T. Toh, K. P. Lim, Y. Yang and D. T. Tan, "Exploring high refractive index silicon-rich nitride films by low-temperature inductively coupled plasma chemical vapor 
deposition and applications for integrated waveguides," ACS App. Mat. Interfaces, vol. 7, no. 39, pp.21884-21889, Sep. 2015.

[29] M. R. Wang Rusli J. L. Xie, N. Babu, C. Y. Li and K. Rakesh, "Study of oxygen influences on carbon doped silicon oxide low $\mathrm{k}$ thin films deposited by plasma enhanced chemical vapor deposition," J. App. Physics, vol. 96, no. 1, pp. 829-834, July 2004.

[30] J. Binner and Y. Zhang, "Characterization of silicon carbide and silicon powders by XPS and zeta potential measurement", Journal of Materials Science Letters 20, 123-126, 2001

[31] J. Tauc, "Optical properties and electronic structure of amorphous Ge and Si," Mater. Res. Bull., vol. 3, no. 1, pp. 37-46, Jan. 1968.

[32] K. Warhoff, A. Driessen, P. V. Lambeck, L. T. H. Hilderink, P. W. C. Linders, Th. J. A. Popma, "Plasma enhanced chemical vapor deposition silicon oxynitride optimized for application in integrated photonics," Sens. and Actuators A: Physical, vol. 74, no. 1, pp. 9-12, Apr. 1999.

[33] R. Germann, H. W. M. Salemink, R. Beyeler, G. L. Bona, F. Horst, I. Massarek, B. J. Offrein, "Silicon oxynitride layers for optical waveguide applications," Jour. Electro. Society, vol. 147, no. 6, pp. 2237-2241, Jun 2000.

[34] F. G. Johnson, O. S. King, J. V. Hryniewicz, L. G. Joneckis, S. T. Chu, D. M. Gill, "Use of deuterated gases for the vapor deposition of thin films for low-loss optical devices and waveguides," US Patent, 6 614, 112, Sep. 2, 2003.

[35] A. Trenti, M. Borghi, S. Biasi, M. Ghulinyan, F. Ramiro-Manzano, G. Pucker and L. Pavesi, "Thermo-optic coefficient and nonlinear refractive index of silicon oxynitride waveguides." AIP Advances, vol. 8, no. 025311, pp. 1-7, Feb. 2018.

[36] D. Melati, A. Melloni and F. Morichetti, "Real photonic waveguides: guiding light through imperfections," Adv. Opt. and Photonics, vol. 6, no. 2, pp. 156-224, Ju. 2014.
[37] F. Morichetti, A. Canciamilla, C. Ferrari, M. Torregiani, A. Melloni, and M. Martinelli, "Roughness induced backscattering in optical silicon waveguides," Phy. Rev. Letters, vol. 104, no. 033902, pp. 1-4, Jan. 2010.

[38] F. Morichetti, A. Canciamilla and A. Melloni, "Statistics of backscattering in optical waveguides," Opt. Letters, vol. 35, no. 11, pp. 1777-1779, Jun. 2010.

[39] L. B. Soldano, and E. C. Pennings, "Optical multi-mode interference devices based on self-imaging: principles and applications," Jour. Lightwave Technology, vol. 13, no. 4, pp. 615-627, Apr. 1995.

[40] M. J. R. Heck, J. F. Bauters, M. L. Davenport, D. T. Spencer, J. E. Bowers, "Ultra-low loss waveguide platform and its integration with silicon photonics," Laser \& Photonics Review, vol. 8, no. 5, Sep. 2014.

[41] M. Belt, M. L. Davenport, J. E. Bowers, and D. J. Blumenthal, "Ultralow-loss Ta2O5-core/SiO2-clad planar waveguides on Si substrates," Optica, vol. no. 5, pp. 532-536, May (2017) 